\title{
Three-Dimensional Reconstruction of Full-Length Tankyrase 1
}

Angelina Chemeris ${ }^{1,2^{*}}$, Alexis M. Gautreau ${ }^{2}$ and Olga S. Sokolova ${ }^{1 *}$

${ }^{1 .}$ Lomonosov Moscow State University, Faculty of Biology, Moscow, Russia.

2. Ecole Polytechnique, Institut Polytechnique de Paris, Palaiseau, France.

*Corresponding authors: sokolova@mail.bio.msu.ru; Angelina1707@mail.ru

Tankyrases are the enzymes that interact with a wide spectrum of proteins and mediate their poly(ADPribosyl)ation (PARylation). Hence, Tankyrases regulate a variety of substantial processes like telomere length regulation, sister chromatid separation, mitosis, glucose metabolism and the Wnt/ $\beta$-catenin signalling pathway [1-2]. Tankyrases were also found to be the signalling targets of mitogen-activated protein kinase MAPK in Golgi [3]. Moreover, PARylation can be involved in the process of ubiquitination and subsequent degradation of PARylated proteins. Most of the binding proteins were PARylated by Tankyrase1/2. Aberrations in PARP activity are coupled with tumorigenesis [4].

Tankyrases comprise of two highly homologous proteins: $142 \mathrm{kDa}$ Tankyrase-1 (TNKS1) and $130 \mathrm{kDa}$ Tankyrase-2 (TNKS2), which are ubiquitously expressed in mammalian cell lines. TNKS1 and TNKS2 are supposed to possess the same functions. Tankyrase-1 is formed by the N-terminal HPS domain, five ankyrin repeat clusters (ARC domains), sterile alpha-motif SAM and a catalytic domain PARP. The SAM domain mediates the oligomerization of Tankyrase 1, which is crucial for its catalytic activity [5]. The ARC domain is responsible for protein-protein interactions. Each ARC domain consists of several ankyrin (ANK) repeats that are responsible for protein-protein interactions [6]. ARC3 showed no activity in protein binding, suggesting that it may play only a structural role [7].

It was previously shown that Tankyrase can fold into a compact structure, thus enabling the interaction between the catalytic PARP domain and the substrates bound to the ARCs region. Moreover, it was shown that ARC3 and ARC4 clusters are separated by a flexible linker. The three domains of Tankyrases were previously crystallized separately [8]. However, full-length tankyrases were never crystallized, most likely because of their flexibility.

In our work we have used Transmission Electron Microscopy (TEM) and negative staining of biological samples, which are able to detect and display distinct transformational states of flexible proteins. The protein was purified from a stable cell line (HEK293 Flp-In T-REx), expressing full-length Tankyrase 1 fused with a $\mathrm{N}$-terminal $\mathrm{PC}$ tag, placed onto electron grids, and then negatively stained with a $1 \%$ uranyl acetate. We obtained the electron micrographs of the prepared grids using a $200 \mathrm{kV}$ electron microscope Jeol 2100. Micrographs (Fig. 1A) enabled us to collect 6500 single particles (some class-averages are shown on the Fig. 1B), which later allowed us to determine the full-length three-dimensional reconstruction of Tankyrase-1 (Fig. 2A). We were able to dock the crystal structures of ARC, SAM and PARP domains into both 3D envelopes (Fig. 2B). In free TNKS1, the fit was good for the ARC and SAM domains and limited for the PARP domain, which appeared to be V-shaped in the electron density. Thus, our 3D reconstruction is the first structural model of a full-length Tankyrase. Our next goal is to study various ligands that can bind TNKS1 and induce its conformational change [9]. 
References:

[1] S Smith et al., Science 5393 (1998) p. 1484.

[2] L Mariotti, K Pollock and S Guettler, British Journal of Pharmacology 174 (2017), p. 4611.

[3] NW Chi and HF Lodish, Journal of Biological Chemistry 275 (2000), p. 38437.

[4] H Wei and X Yu, Genomics, Proteomics and Bioinformatics 14 (2016), p.131.

[5] AA Riccio et al., Structure 24 (2016), p.1573.

[6] SG Sedgwick and SJ Smerdon, Trends in Biochemical Science 24 (1999), p. 311.

[7] S Guettler et al., Cell 147 (2011), p. 1340.

[8] T Eisemann et al., Structure 24 (2016), p. 1679.

[9] Authors acknowledge the funding from RSF (\#19-14-00168 to O.S.S.). A.C. has been supported in part by Vernadski program from the French Embassy in Moscow, Russia.

A

B
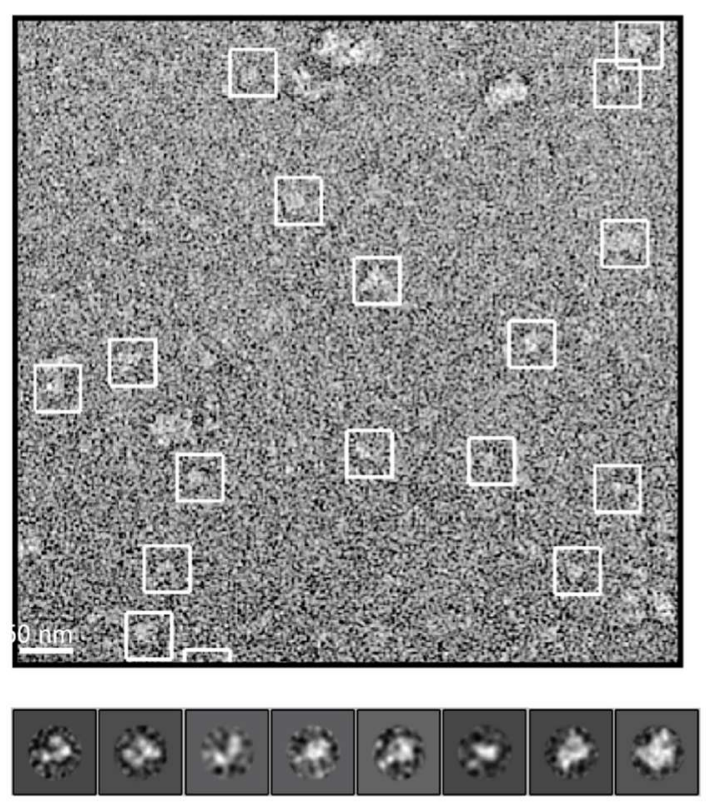

TNKS1
A

B
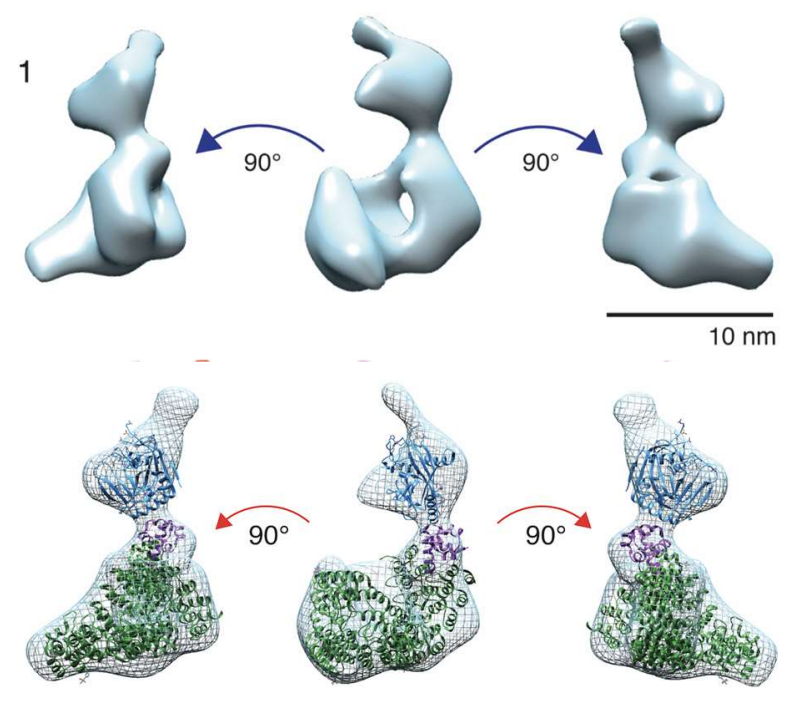

Figure 2. 3D reconstruction of TNKS1. The three views differ from each other by rotations of 90 degrees. (B) Docking of the crystal structures of PARP domain (blue), SAM domain (fuchsia) and the 5 Ankyrin Repeats Clusters (green) into the electron density model of TNKS1. 